\title{
Comunicação Serial para um Simulador de Imersão de Carrinho de Rolimã, Comparando JSON, XML e Texto
}

\author{
Tiago Guerino de Oliveira Bassani ${ }^{1}$, Kleber de Oliveira Andrade ${ }^{1}$
}

${ }^{1}$ Faculdade de Tecnologia de Americana Ministro Ralph Biasi

$$
\text { Americana - SP - Brasil }
$$

\begin{abstract}
In order for the roller car immersion simulator to interact with its controls, the use of serial communication is indispensable. This work aims to address the need to establish a connection through existing message formats. For this it was used an Arduino in communication with a telemetry system developed in Java in order to understand which format is more agile and that has no noticeable delays to users of the simulator.
\end{abstract}

Resumo. Para que o simulador de imersão do carrinho de rolimã possa interagir com seus controles, o uso da comunicação serial é indispensável. Esse trabalho visa atender a necessidade de estabelecer uma conexão através de formatos de mensagens existentes. Para isso foi utilizado de um Arduino em comunicação com um sistema de telemetria desenvolvido em Java a fim de entender qual formato é mais ágil e que não tenha atrasos perceptiveis aos usuários do simulador.

\section{Introdução}

Um carrinho de rolimã ou carrinho de rolamentos é um carrinho construído, geralmente de madeira e rolamentos de aço para suas rodas, utilizados para se correr em ladeiras abaixo. Seu nome é devido ao fato de suas rodas serem rolamentos [Frederico et. al. 2016]. A figura 1 demonstra um exemplo de carrinho de rolimã.

O presente trabalho trata-se de uma pesquisa aplicada. Os resultados obtidos e analisados podem ser empregados em outros simuladores e jogos com controles mais aprimorado. O objetivo do trabalho é analisar diferentes formatos de mensagem enviados através de uma comunicação serial, com fio e sem fio, para controles de direção e freio em um simulador imersivo de carrinho de rolimã.

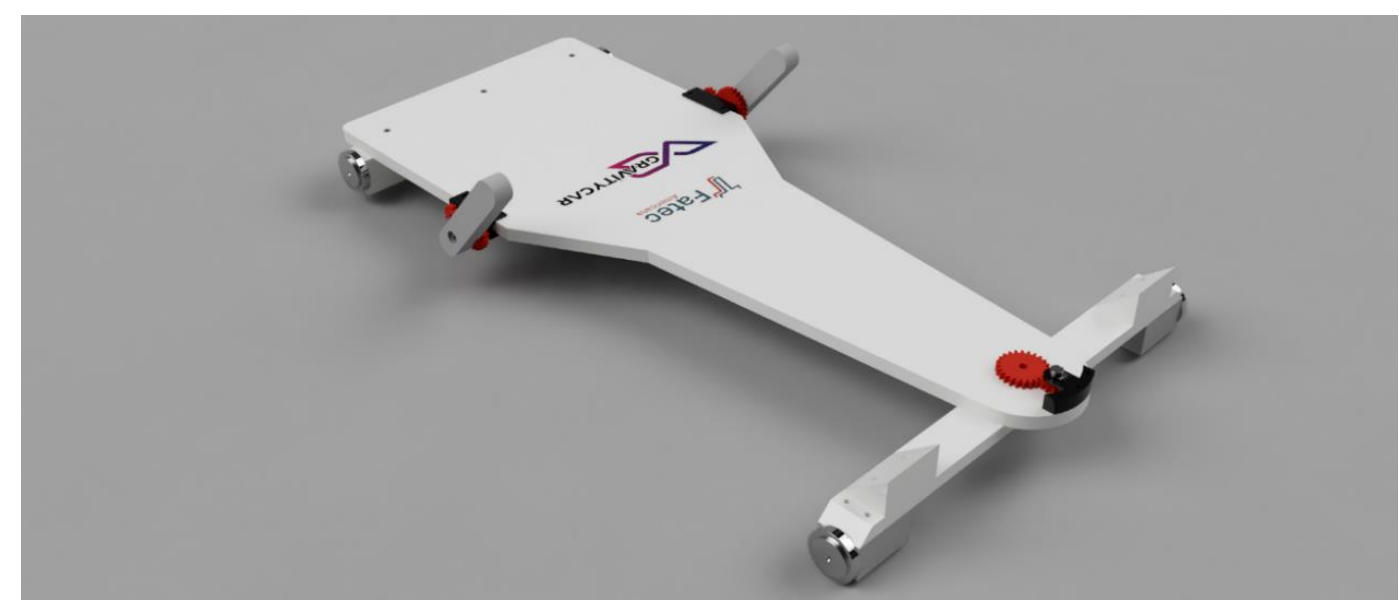

Figura 1. Exemplo de carrinho de rolimã

De acordo com [Banks et al. 2009] simulação é uma imitação de uma operação realizada no mundo real, sendo utilizada junto ao computador ou não. Simulações 
envolvem a gerações de histórias artificiais e observar como essa história interfere na operação do sistema real.

Para que o simulador de imersão de carrinho de rolimã possa ser controlado de maneira que não ocorra atrasos perceptíveis, uma comunicação serial será estabelecida para otimização do tempo de transferência. O formato de mensagem escolhido para que a comunicação ocorra é de suma relevância para que o tempo de resposta entre os dispositivos seja ágil, integro e uniforme. A comunicação serial é empregada em diferentes meios de comunicação entre dispositivos distintos, por exemplo, o envio de uma foto em celular para um computador ou ainda leitores de códigos de barra em um supermercado.

A importância de um sistema que interprete os dados da comunicação serial é muito alta pois sem essa comunicação o simulador não funcionaria de maneira correta e apresentaria atrasos perceptíveis aos usuários.

\section{Revisão Bibliográfica}

Nesta seção será abordado o conceito de comunicação serial, bluetooth, os formatos de mensagem utilizados para as realizações dos testes e as métricas utilizadas.

\subsection{Comunicação Serial}

A comunicação entre dois dispositivos computacionais é possível através de uma interface serial. A comunicação serial é a transferência de dados feita bit a bit. [Rabello 2009]

Rabello [2009] também diz que cada byte enviado ou recebido, na comunicação serial, é feito um bit por vez, e que esses podem assumir valores lógicos ligado (1) ou desligado (0). A velocidade com que esses bits trafegam é medida em bits-por-segundo (bps) ou em em baud rate, que correspondem a quantidade de bits que são enviados ou recebidos em um segundo. Nos computadores, a velocidade de tráfego de bits pode alcançar 19,2kbps ou mais.

\subsubsection{Bluetooth}

A tecnologia Bluetooth foi criada pela L.M.Ericsson em 1994. O nome Bluetooth vem do rei da Dinamarca, Harald Blaatand, que traduzido para o inglês seria Harald Bluetooth, que unificou os reinos da Dinamarca e Noruega. Em 1998 as maiores empresas de tecnologia e telefones fundaram a Bluetooth Special Interest Group (SIG), que hoje conta com mais de 1900 empresas [Zeadally et al. 2019].

O Bluetooth é um meio de comunicação sem fio (inglês Wireless) que opera à curtas distancias na faixa de $2,400 \mathrm{GHz}$ a $2,485 \mathrm{GHz}$, com canais de rádio frequência espaçados a cada $1 \mathrm{MHz}$ [Zeadally et al. 2019].

Duas topologias de conexão são estabelecidas por Bluetooth, piconet e scatternet. A primeira consiste em um dispositivo mestre (master) e um ou mais dispositivos escravos (slaves), com a frequência de operação da piconet definida pelo dispositivo master. A segunda topologia é uma junção de duas ou mais piconets ocupando o mesmo espaço, simultaneamente. Em uma scatternet um dispositivo pode operar como slave em várias piconets, porém apenas em uma como master [Lee et al. 2007].

\subsection{JSON}


JSON (JavaScript Object Notation) é um tipo de armazenamento e transmissão de dados no formato de texto, independente de linguagens. É baseado no formato da estrutura de um objeto Javascript como descrito [Zunke e D'Souza 2014].

De acordo com o ECMA [2017] pode ser composto por apenas nome e valor ou uma coleção de valores, como um array, lista ou vetor. O nome deve ser uma cadeia de caracteres (string) e os valores podem ser booleanos (true, false), numéricos, nulos (null), strings ou arrays.

A estrutura do JSON é composta por um caractere de chave de abertura (" "\{“) e um para fechamento (")"), cada atributo segue-se o uso de dois pontos ("“:") e o valor referente a esse atributo. No caso de mais atributos, deve se empregar a virgula (“",) para separá-los, conforme apresentado na figura 2. O ECMA [2017] esclarece que, no uso de arrays aplica-se o colchete ("[") de abertura e fechamento ("]"), seus valores são separados por vírgula (“,”).

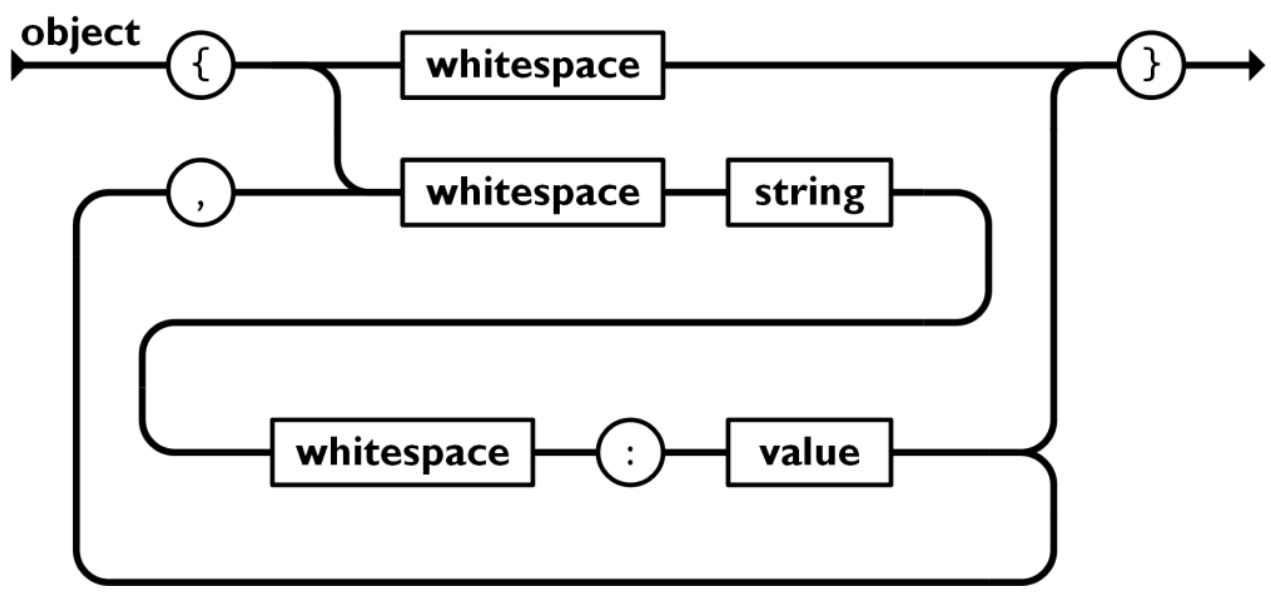

Figura 2. Estrutura JSON

\subsection{XML}

Extensible Markup Language (XML) é um conjunto de regas publicado pelo Wide Wolrd Web Consortium (W3C), grupo que regulamenta os padrões utilizados na internet. XML foi desenvolvido para facilitar a execução e comunicação com SGML e HTML [Zunke e D'Souza 2014].

O W3C define que documento $X M L$ contenha um ou mais elementos separados por marcações (tag) de início, fim e tag vazia. As tags de início são compostas por sinal de menor ("<") seguido do nome da tag, pode ter ou não atributos, e o sinal de maior (">>) indicando o fim da tag. As tags de fim devem conter sinal de menor seguido de uma barra (“"”) esta indica fechamento, repetir o nome da tag e o sinal de maior. Já as tags vazias são exatamente como as de início, porém antes do sinal de maior, se tem uma barra de fechamento, conforme mostra a figura 3 [Consortium 2008].

\subsection{Métricas}

Para uma análise melhor dos resultados foi feito o uso de média aritmética simples e desvio padrão. 


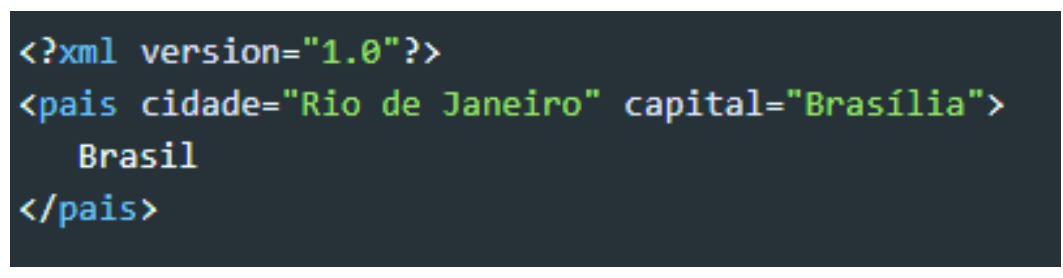

Figura 3. Estrutura XML

Média aritmética simples é a média mais simples de ser calculada. Conforme a equação 1 a média aritmética simples $\bar{x}$ é dada por uma lista com $n$ elementos, sendo $n>$ 1, basta somar seus elementos $x$ e dividir pela quantidade $n$ [Pereira 2014].

$$
\bar{x}=\frac{1}{n} \sum_{i-1}^{n} x_{i}
$$

\section{Equação 1. Média aritmética simples}

Segundo Lunet [2006] desvio padrão representa a variação entre as médias calculadas. A equação 2 representa o cálculo do desvio padrão. O resultado $D p$ é obtido por meio de uma raiz quadrada da soma de uma lista com $n$ valores, tendo o valor de cada elemento $x$ subtraído da média aritmética $\bar{x}$ elevado ao quadrado e dividido por $n$

$$
D p=\sqrt{\frac{\sum_{i-1}^{n}\left(x_{i}-\bar{x}\right)^{2}}{n}}
$$

\section{Equação 2. Desvio padrão}

\section{Materiais e métodos}

O conteúdo desta seção trata de listar os materiais elétricos, ferramentas utilizadas na codificação e métodos para elaboração do sistema de telemetria

\subsection{Eletrônica}

Durante a elaboração dos controles do simulador utilizou-se os seguintes componentes eletrônicos: um Arduino Uno para a leitura dos valores; três potenciômetros B20K que geram os valores; um cabo USB (Universal Serial Bus) tipo AB para a compilação do programa do Arduino e comunicação; e um módulo bluetooth HC-05 para envio de dados do hardware ao software, conforme mostrado no esquema elétrico na figura 4.

Arduino é uma plataforma open-source para desenvolvimento e que facilita a comunicação entre hardware e softwares. Tem uma capacidade de comunicação com vários tipos de componentes como: LEDs, sensores, motores entre outros tipos. A interação entre eles é feita através da linguagem de programação própria que se assemelha as linguagens $\mathrm{C} / \mathrm{C}++$ [McRoberts 2011].

Potenciômetro é um componente eletrônico cuja função é variar sua resistência elétrica através de um eixo giratório ou deslizante. O movimento do eixo faz com que um contato deslize sobre uma superfície resistiva como grafite. Potenciômetros são muito utilizados na eletrônica como em controles de volume, sensibilidade entre outros [Braga 2012]. 


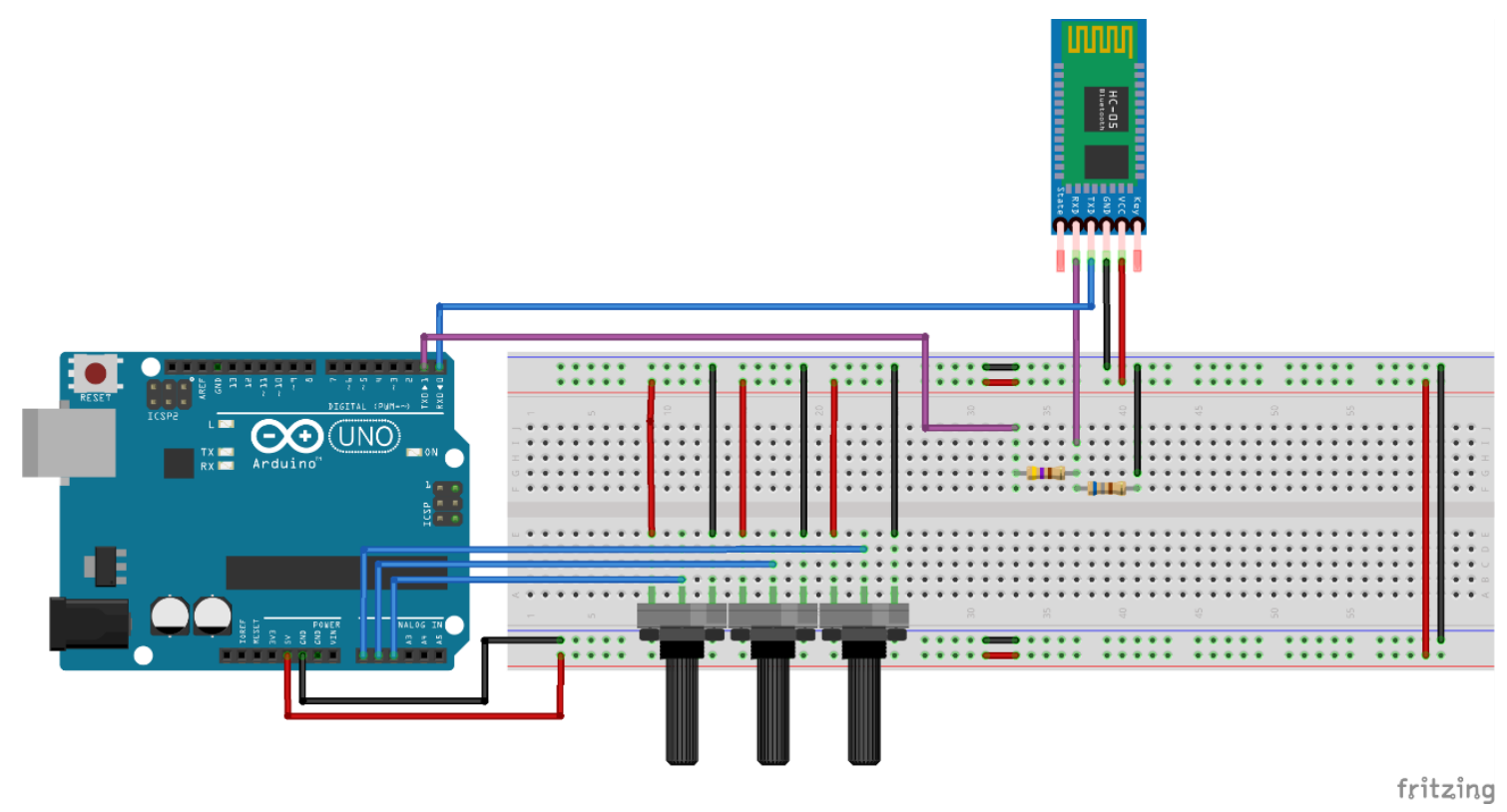

Figura 4. Esquema elétrico

\subsection{Ferramentas}

Elaborou-se a programação do Arduino para a leitura dos potenciômetros e envio de mensagens. Esta programação realizou-se no ambiente de desenvolvimento integrado (IDE - Integrated Development Environment) Arduino IDE 1.8.10, que está disponível para download no site arduino.cc. Nessa etapa foi utilizado a biblioteca ArduinoJson em sua versão 6.11.0, disponível através no Arduino IDE para download, empregada na serialização de $J S O N$.

Para que haja a leitura dos valores transportados pela porta serial houve a necessidade de desenvolver um sistema de telemetria em Java. A IDE utilizada para o desenvolvimento foi o NetBeans juntamente com o repositório Maven, uma plataforma que disponibiliza bibliotecas à serem aplicadas em projetos Java. Todas as bibliotecas utilizadas neste projeto estão disponíveis no Maven, exceto ArduinoJson, que está disponível na Arduino IDE.

Bibliotecas adicionadas ao projeto são: JSerialComm, faz com que os valores indicados no Arduino possam ser interpretados pelo sistema; JFreeChart é utilizada para a criação do gráfico telemétrico dos valores encontrados nos potenciômetros; JSON-java realiza serialização e desserialização do JSON enviado através da porta serial ao sistema Java pelo Arduino e vice-versa; XStream serve como serialização e desserialização do $X M L$ aplicado para comparativo de velocidade entre as mensagens.

\subsection{Sistema Telemétrico}

A codificação do sistema telemétrico ocorreu através da IDE NetBeans criando-se uma janela com botões de conexão, caixas de combinação para a seleção da porta serial e baud rate, três painéis onde serão exibidos os gráficos e cinco caixas de texto que exibe os valores de cada um dos três potenciômetros: valores mínimo, médio e máximo na cor preto; atual na cor azul; média móvel na cor vermelho. Em seguida foi feita a implementação das classes MovingAverange e StaticsTracker conforme consta no diagrama 1

Conforme o diagrama 2 foi construído a classe RealTimeLineChart para gerar o gráfico de linha em tempo real, a fim de facilitar a visualização da variação dos valores 
obtidos pelos potenciômetros. A leitura do gráfico se dá por duas linhas nas cores azul e vermelho que representam os valores atual e média móvel respectivamente.

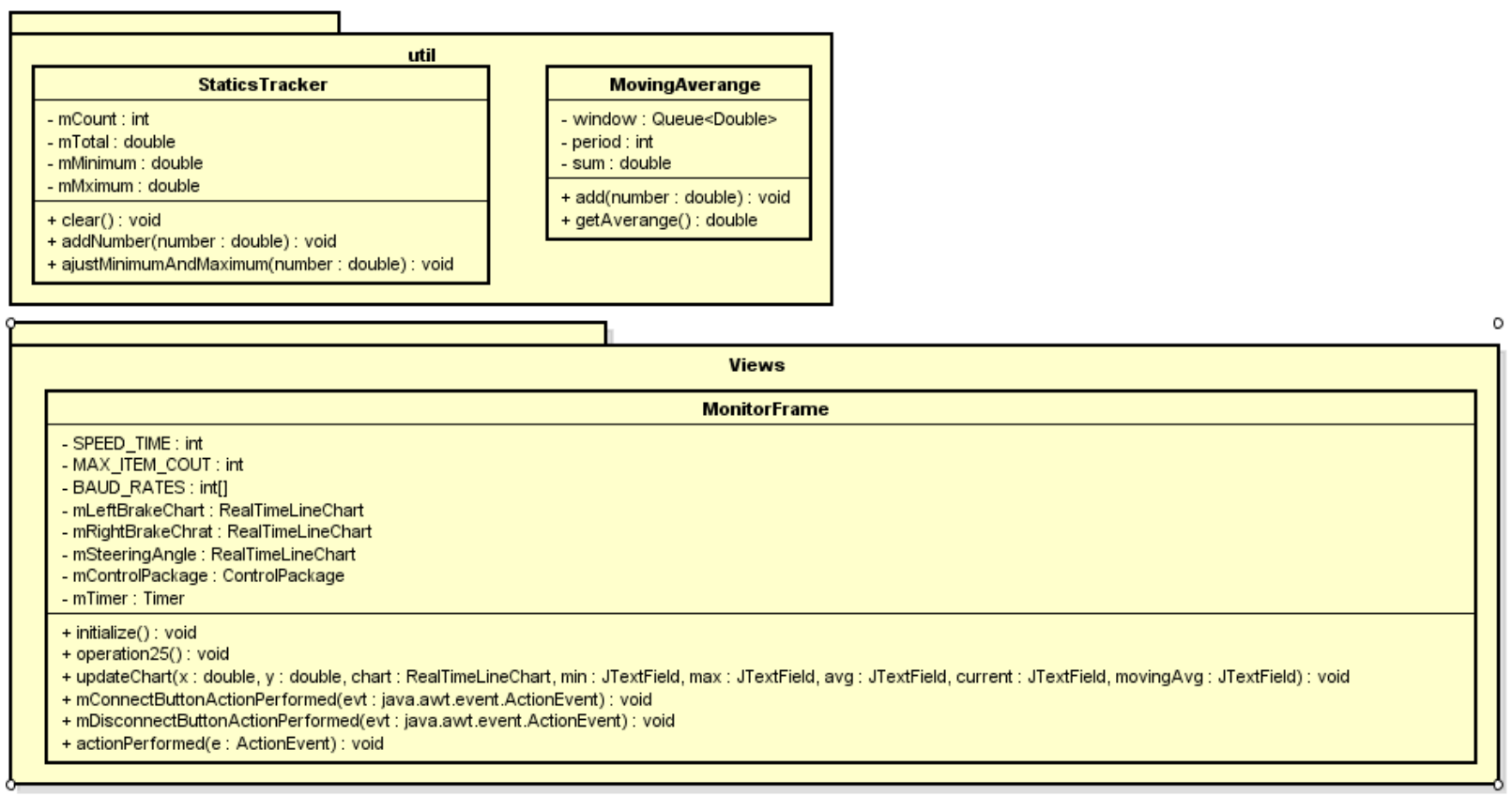

\section{Diagrama 1. Diagrama de Classes 1}
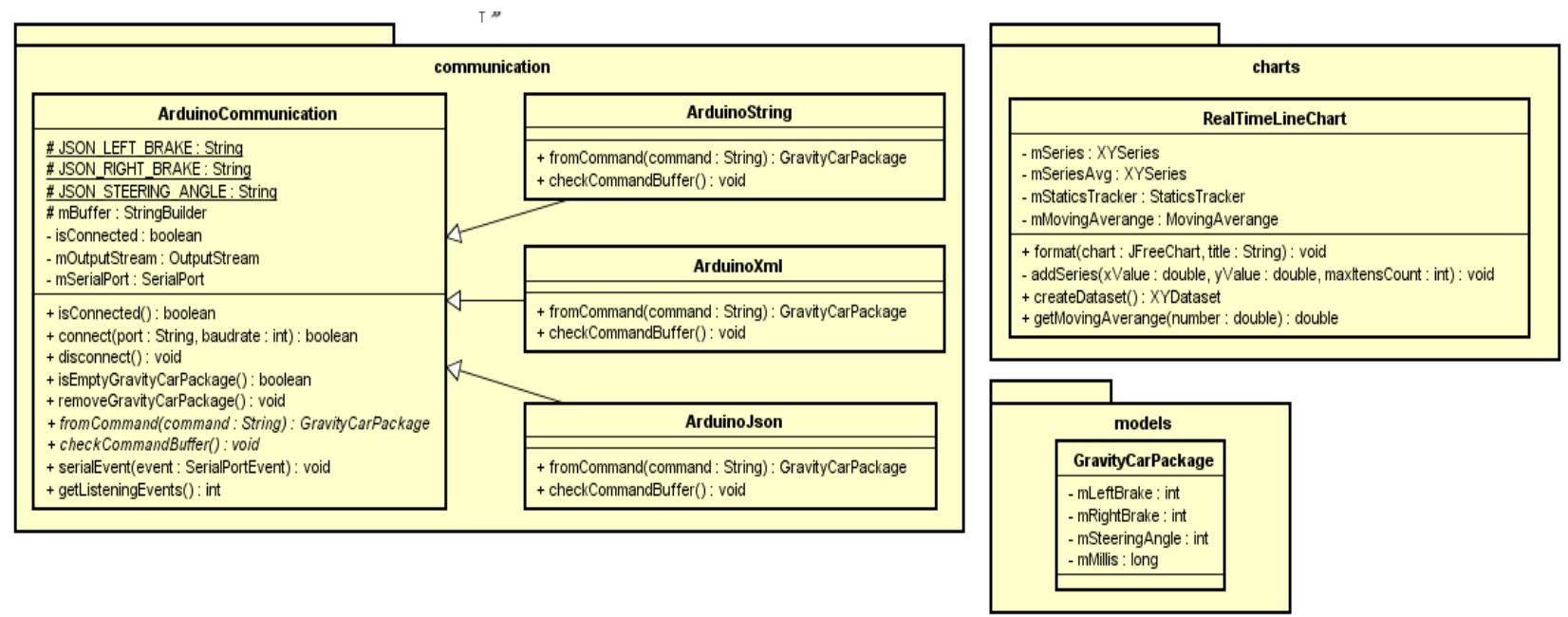

Diagrama 2. Diagrama de classes 2

A classe abstrata ArduinoCommunication, com a finalidade de transmitir dados através da porta serial. Os métodos fromCommand e checkCommandBuffer são abstratos para assim serem implementados após a escolha do formato de mensagem. Devido a isso três classes tiveram que implementar os métodos abstratos. Na ArduinoJson foi importado a classe $J_{S o n-j a v a}$ para desserialização do $J S O N$ enviado pelo Arduino. Já na classe ArduinoXml, utilizou-se da biblioteca XStream para desserializar o $X M L$ enviado. A comunicação entre o sistema desktop e o Arduino se deu por meio da comunicação serial, através do cabo USB e do Bluetooth. 


\section{Resultados}

A seguir serão mostrados as configurações e resultados obtidos pelo experimento, fazendo o uso das métricas estabelecidas.

\subsection{Configurações}

O experimento de envio de mensagens ao sistema telemétrico foi realizado em um processador Intel Core i5-7200U, com memória de 8GB e sistema operacional Windows 10 .

Ao todo foram mil e vinte (1020) repetições para cada teste de mensagem enviado do Arduino ao sistema de telemetria através da porta serial por meio do bluetooth ou USB. A mensuração dos resultados dos tempos de resposta entre cada formato de mensagem realizada, se deu em comparação das equações matemáticas de média e desvio padrão. Assim pode-se analisar qual formato de mensagem mais adequado, pois seu tempo de resposta será o menor.

Nos testes foram utilizados três formatos de mensagem, JSON, XML e texto. Cada mensagem foi enviada com o conteúdo a palavra "testel", que ao ser entregue no sistema de telemetria retornava ao Arduino a mesma mensagem e fazia o cálculo de diferença entre o horário de envio e o horário de recebimento em milissegundos.

\subsection{Métricas}

Nesta etapa foi executado o teste de performance entre JSON, XML e texto. As mil e vinte mensagens tiveram seu horário de recebimento armazenado em uma lista através da função do Java Localtime.now() em milissegundos (ms). O tempo entre as mensagens foi calculado subtraindo o horário da mensagem recebida do horário de recebimento da mensagem anterior. Em seguida é feito o cálculo de média aritmética simples e desvio padrão.

\subsection{Discussão}

O gráfico 1 apresenta os resultados nos testes para os valores dos formatos de mensagem. Como observado na coluna azul os formatos apresentam grande diferença entre si. Pode se observar que o JSON apresenta o tempo de envio entre as mensagens menor que $X M L$ e texto, sendo aproximadamente $31,5 \mathrm{x}$ mais rápido que o $X M L$ e aproximadamente $28,3 \mathrm{x}$ mais veloz que o texto. Analisando o desvio padrão, podemos constatar que o JSON também possui maior eficácia na variação dos tempos com relação aos demais.

Observado também na coluna laranja que o JSON também apresenta valores muito superiores as outras opções de mensagens enviadas. É cerca de 13,1x e 12,4x mais rápida que $X M L$ e texto respectivamente.

A partir dos resultados obtidos pelas equações matemáticas e em comparação as colunas do gráfico, pode se analisar e optar pelo formato de mensagem e meio de comunicação usada no simulador de imersão. Os valores dos potenciômetros, lidos pelo Arduino, serão enviados através de $J S O N$ por meio de uma comunicação serial estabelecida por Bluetooth. A escolha da conexão levou em consideração, além da velocidade, a praticidade e conexão com outros tipos de dispositivos, por exemplo, dispositivos móveis. Assim com a definição de ambos já citados concluiu-se o sistema telemétrico conforme mostrado na figura 5.

O Sistema de telemetria apresentado na figura 5 consiste em apresentar em formas de gráficos de linhas os valores obtidos pelos potenciômetros de ângulo de esterço, freio direito e freio esquerdo de maneira independente. As linhas azuis correspondem aos 
valores lidos pelo Arduino e as linhas vermelhas os valores depois de aplicado o filtro de média móvel.

\section{Conclusão}

Ao final do comparativo de velocidade apresentado conseguiu-se realizar a análise e consequente escolha do formato de mensagem mais adequado a realidade do simulador de imersão de carrinho de rolimã. O formato te mensagem escolhido para os controles de freio e direção do simulador foi formato $J S O N$, que apresenta velocidade superior aos outros formatos analisados, conforme o gráfico 1. O meio de comunicação bluetooth, apesar não ser o mais veloz quando envia a mensagem em JSON, possibilitará a portabilidade do simulador em diversos dispositivos, como celular e óculos de realidade virtual

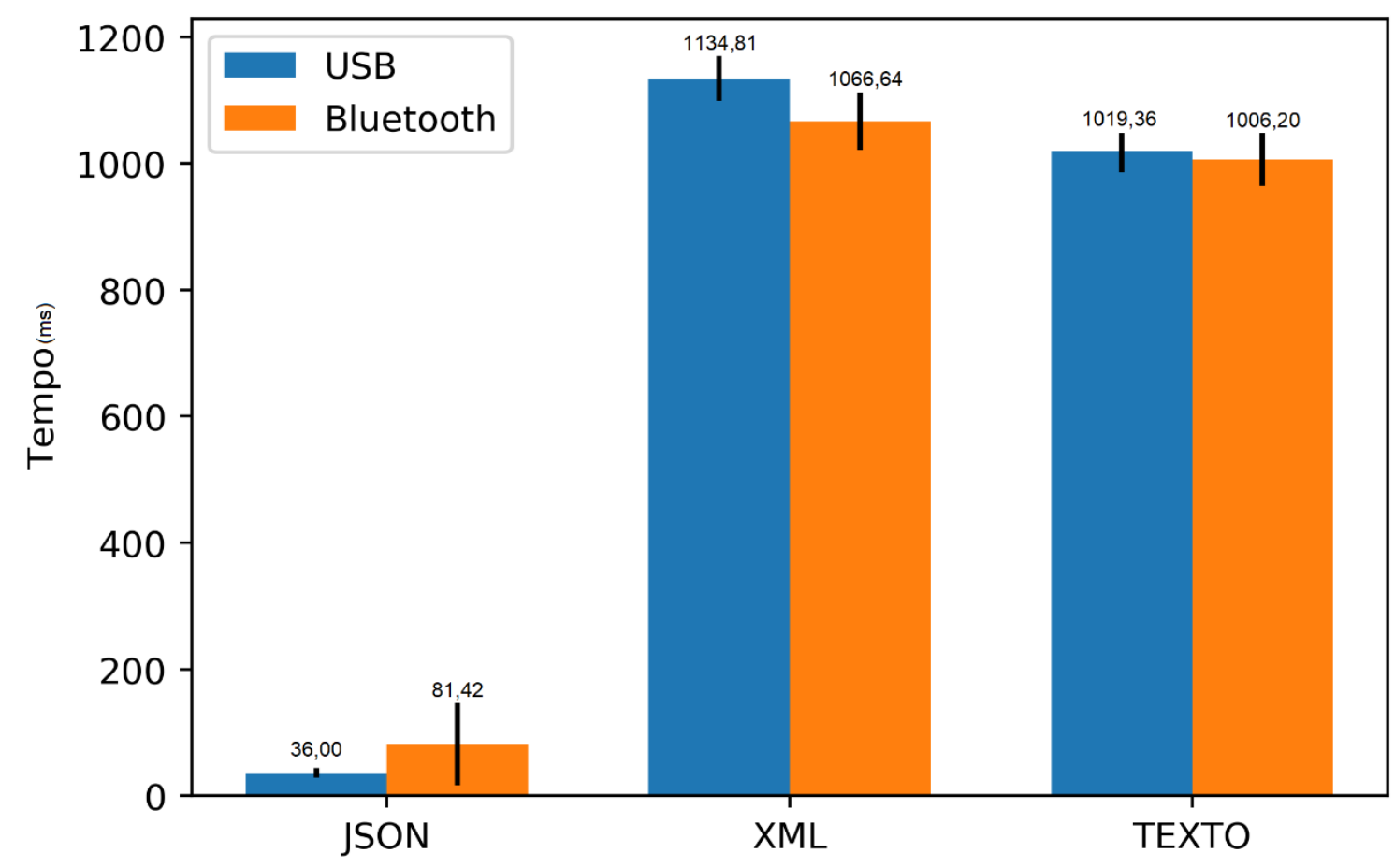

Gráfico 1. Velocidade de transmissão

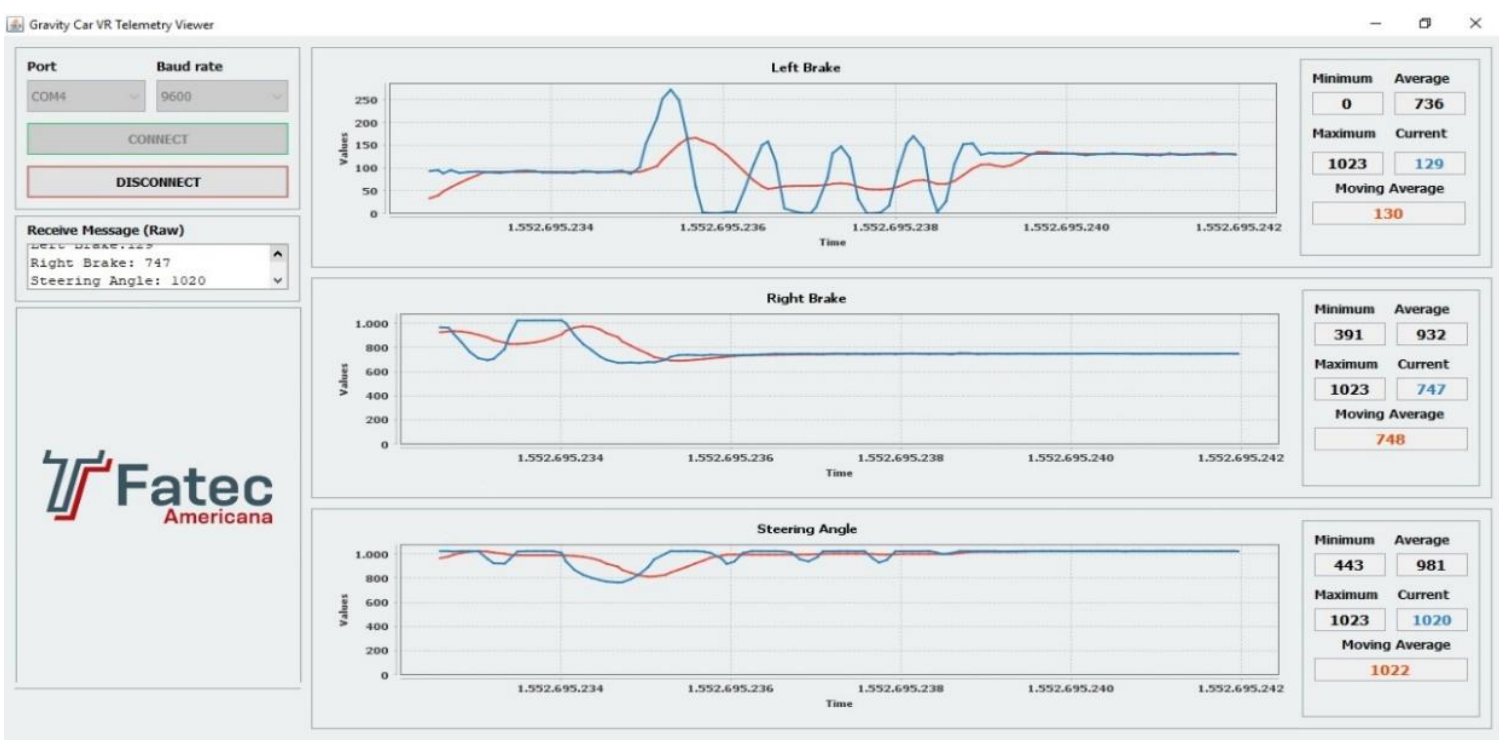

Figura 5. Sistema de telemetria 
Para trabalhos futuros, deverá ser feito um comparativo utilizando uma rede de internet, por meio $\mathrm{Wi}-\mathrm{Fi}$ e por cabo Ethernet, para assim se ter uma melhor avaliação dos dados e struct e Binary JSON (BSON) para formatos para o envio das mensagens. Para o sistema de telemetria controles para motores de vibração afim de proporcional melhor experiencia ao usuário do simulador.

\section{Referencias}

Banks, J., CarsonII, J. S., and Nelson, B. L. (2009). Discrete-Event System Simulation. Pearson.

Braga, N. C. (2012). Conserte Tudo: Guia Prático para Reparador Eletronico. NBC, São Paulo - Brasil, first edition.

Consotium, W. W. W. (2008). Extensible Markup Language (XML) 1.0. Quinta Edição. Disponível em: <https://www.w3.org/> Acesso em: 13 nov. 2019.

ECMA (2017). Stardard ECMA-404: The JSON Data Interchange Syntax. Disponível em: $\quad<$ https://www.ecma-international.org/publications/files/ECMA-ST/ECMA404.pdf>. Acesso em 14 out, 2019.

Frederico, A. S., Toledo, G. P., Barros, M. V. de V., Benini, L. (2016). Processo de fabricação de um carrinho de rolimã. Eu, a Industria e o Mundo.

Lee, J.-S., Su, Y.-W., and Shen, C.-C. (2007). A comparative study of wireless protocols: Bluetooth,uwb,zigbee,andwi-fi. The 33rd Annual Conference of the IEEE Industrial Electronics Society (IECON).

Lunet, N., Severo, M., and Barros, H. (2006). Desvio padrão ou erro padrão. ArquiMed.

McRoberts, M. (2011). Arduino Básico. Novatec.

Pereira, J. D. C. (2014). Médias: Aritmética, geométrica e harmonica. Tese de mestrado, Universidade Estadual de Campinas.

Rabello, L. M. (2009). Programa em linguagem java para comunicação serial. Comunicado técnico 109.

Zeadally, S., Siddiqui, F., and Baig, Z. (2019). 25 years of bluetooth technology. Future Internet. DOI:10.3390/fi11090194

Zunke, S. and D'Souza, V. (2014). Json vs xml: A comparative performance analysis of data exchange formats. IJCSN International Journal of Computer Science and Network. 